\title{
Avaliação da deglutição e o impacto na qualidade de vida em pacientes com doença de parkinson
}

\author{
Evaluation of deglutition and the impact on the quality of life of patients with parkinson's \\ disease
Evaluación de la deglutición y el impacto en la calidad de vida en pacientes con enfermedad de parkinson

Ana Larisse Gondim Barbosa ${ }^{1 *}$, Gisele Vieira Hennemann Koury ${ }^{1}$, Felipe Xavier de Souza ${ }^{1}$, Jocyane de Souza Andrade ${ }^{1}$, André dos Santos Brandão ${ }^{1}$, Igor Isamu Couceiro Seto ${ }^{1}$, Fabíola Milenne Moraes e Silva ${ }^{1}$, Mariângela Moreno Domingues ${ }^{2}$, Niele Silva de Moraes ${ }^{2}$.

\section{RESUMO}

Objetivo: Avaliar a presença de disfagia e seu impacto na qualidade de vida de pacientes portadores de doença de Parkinson (DP), correlacionando o estadiamento da doença, a severidade da disfagia e a auto avaliação do risco de disfagia. Métodos: Estudo observacional e transversal, avaliando 24 pacientes portadores de DP através da escala para sintomas motores de Hoenh e Yahr, da avaliação endoscópica da deglutição (VED), de questionário sobre a qualidade de vida (The 39 item Parkinson's Disease Question PDQ-39) e de um instrumento de auto avaliação do risco de disfagia e aspiração (Eating Assessment Tool EAT-10). Resultados: Na VED, $87,5 \%$ dos pacientes apresentaram disfagia, com gravidade independente da severidade motora da doença. Não houve significância entre a gravidade da disfagia e a auto avaliação de disfagia e aspiração. No PDQ-39 houve pior percepção sobre a qualidade de vida com o avançar da doença $(\mathrm{p}=0,003)$, com significância no domínio da cognição nos disfágicos graves. Conclusão: a disfagia é um sintoma comum e independente da severidade motora da DP, devendo ser investigada e reabilitada por equipe multiprofissional precocemente a fim de reduzir o impacto na qualidade de vida destes pacientes.

Palavras-chaves: Doença de Parkinson, Transtornos de deglutição, Qualidade de vida.

\begin{abstract}
Objective: To evaluate the presence of dysphagia and its impact in the quality of life of patients with Parkinson's disease (PD), correlating the staging, severity of dysphagia and self-assessment of dysphagia risk. Methods: An observational and cross-sectional study evaluating 24 patients with PD through the Hoenh and Yahr motor symptoms scale, the endoscopic swallowing assessment (VED), and the quality of life questionnaire (The 39 item Parkinson's Disease Question - PDQ-39) and a self-assessment tool for risk assessment of dysphagia and aspiration (EAT-10). Results: In VED, 87.5\% of the patients presented dysphagia, with severity regardless of the motor severity of the disease. There was no significance between dysphagia severity and dysphagia and aspiration self-assessment. In PDQ-39, there was a worse perception of quality of life as the disease progressed $(p=0.003)$, with significance in the domain of cognition in severe dysphagic patients. Conclusion: dysphagia is a common symptom independent of the motor severity of PD and should be investigated and rehabilitated by a multidisciplinary team early in order to reduce the impact on the quality of life of these patients.
\end{abstract}

Keywords: Parkinson's disease, Swallowing disorders, Quality of life.

\footnotetext{
${ }^{1}$ Hospital Universitário Bettina Ferro de Souza (HUBFS), Universidade Federal do Pará (UFPA), Belém-PA.

*E-mail: analarisse@gmail.com

2 Universidade Estadual do Pará (UEPA), Belém-PA.
} 


\section{RESUMEN}

Objetivo: evaluar la presencia de disfagia y su impacto en la calidad de vida de los pacientes con la enfermedad de Parkinson (EP), correlacionando la estadificación de la enfermedad, la gravedad de la disfagia y la autoevaluación del riesgo de disfagia. Metodos: un estudio observacional y transversal que evaluó a 24 pacientes con EP a través de la escala de síntomas motores de Hoenh y Yahr, la evaluación de deglución endoscópica (VED) y el cuestionario de calidad de vida (La pregunta de la enfermedad de Parkinson de 39 ítems - PDQ-39) y una herramienta de autoevaluación para la evaluación del riesgo de disfagia y aspiración (EAT-10). Resultados: en VED, el $87.5 \%$ de los pacientes presentaron disfagia, con la severidad independientemente de la gravedad motora de la enfermedad. No hubo significación entre la gravedad de la disfagia y la autoevaluación de disfagia y aspiración. En PDQ-39, hubo una peor percepción de la calidad de vida a medida que la enfermedad progresó $(p=0.003)$, con importancia en el dominio de la cognición en pacientes con disfagia severa. Conclusión: la disfagia es un síntoma común independiente de la gravedad motora de la EP, y debe ser investigada y rehabilitada por un equipo multidisciplinario temprano para reducir el impacto en la calidad de vida de estos pacientes.

Palabras clave: Enfermedad de Parkinson, Trastornos de la deglución, Calidad de vida.

\section{INTRODUÇÃO}

A Doença de Parkinson (DP), descrita inicialmente pelo médico inglês James Parkinson em 1817, é uma desordem neurodegenerativa progressiva do sistema nervoso central que ocorre devido à morte de células da substância negra compacta e de outros núcleos pigmentados do tronco encefálico, produzindo um esgotamento seletivo do neurotransmissor dopamina, importante no controle dos movimentos. A DP é um dos distúrbios neurológicos mais comum no mundo, caracterizada por afetar principalmente o sistema motor (PRINGSHEIM T, et al., 2014).

Os sintomas e a evolução da doença são insidiosos no decorrer dos anos. Surgem entre 40 e 70 anos e seu pico de início se manifesta na sexta década de vida, sendo os homens mais acometidos que as mulheres (PRINGSHEIM T, et al., 2014; GASPARIM AZ, et al., 2011). Nos estágios iniciais da doença, os sinais mais evidentes são os tremores, rigidez, lentidão dos movimentos e a dificuldade em caminhar (CARNEIRO D, et al., 2013). Nos estágios tardios, são frequentes sintomas não motores, como depressão, ansiedade e demência. Dentre os sintomas não motores, observa-se a disfagia, que é a dificuldade no processo de deglutição dos alimentos, um sintoma comum na DP, podendo se desenvolver em qualquer momento da evolução da doença (LIM A, et al., 2008; HELY MA, et al., 2008; GASPARIM AZ, et al., 2011).

Com o envelhecimento da população mundial e o exponencial surgimento de doenças neurodegenerativas, o cenário da saúde pública moderna tem sido modificado. Estima-se que em 2030 quase nove milhões de pessoas sofrerão de DP no mundo (DORSEY ER, et al., 2007), fato já observado na atenção secundária e terciária que vem recebendo uma demanda cada vez maior de pacientes que requerem cuidados redobrados por consequências fisiológicas ou comorbidades do envelhecimento associadas a doenças degenerativas como a DP.

A dificuldade no processo de deglutição dos alimentos na DP é devida à inabilidade da realização rápida e coordenada dos movimentos envolvidos nesse processo (GASPARIM AZ, et al., 2011). Em decorrência da rigidez muscular e da bradicinesia, as manifestações disfágicas podem ocasionar atraso no reflexo da deglutição e mobilidade reduzida das estruturas orofaríngeas, com possibilidade de perda prematura de alimento e o seu acúmulo na parte oral da faringe, valéculas epiglóticas (VE) e recessos piriformes (RP), o que dificulta a ingestão dos medicamentos, leva a desnutrição e favorece a aspiração pulmonar, que é uma das principais causas de morte na DP (SUTTRUP I e WARNECKE T, 2015).

A broncoaspiração acontece em cerca de $50 \%$ dos doentes com DP e conduz a um elevado risco de pneumonia de aspiração e morte nesta população (SANTOS HIV, 2017). O conhecimento das alterações 
disfágicas em paciente com DP é decisivo para a manutenção da qualidade de vida (MONTEIRO D, et al., 2014). A disfagia pode ser avaliada através de diversos instrumentos. Os mais relevantes na prática clínica são a videofluoroscopia, considerada padrão ouro, e a nasofibrolaringosocopia para avaliação da deglutição, também chamada de videoendoscopia da deglutição (VED).

Apesar da videofluoroscopia ser considerada padrão ouro na avaliação da disfagia, há limitações no seu uso, devido a exposição a radiação, necessidade de aparelhos de radioscopia, restrição do diagnóstico de lesões estruturais pequenas (como câncer glótico em fase inicial) e funcionais (como paralisia de pregas vocais). A videofluoroscopia deve ser realizada por radiologista acompanhada por fonoaudiólogo ou por otorrinolaringologista, tem uso importante em pacientes pouco colaborativos, com alto risco de aspirações de pequeno volume e com alterações na fase oral e esofágica presumidas, os quais tem menor sensibilidade diagnóstica com a VED. Em contrapartida, a VED, que é realizada pelo médico otorrinolaringologista e pode ser acompanhado pelo fonoaudiólogo, é simples, barata e pouco invasiva, sem efeito colateral significativo (TSUJI DH, 2017) não necessitando de oferecimento de contraste ou radiação, avaliando de maneira sensível e específica a estrutura e mobilidade faringolaringea, vantagens importantes na comparação com a videofluoroscopia (BELO C, et al., 2009).

Existem ainda testes de triagem para disfagia em pacientes com DP, de simples execução, com perguntas dirigidas para problemas de deglutição e questionários de autoavaliação quanto ao risco de disfagia (GONÇALVES MIR, et al., 2013).

Por se tratar de uma doença crônica incurável, acredita-se que a disfagia possa reduzir a qualidade de vida e aumentar as comorbidades nutricionais e infecciosas, alterando o bem-estar biopsicossocial dos pacientes com DP. Dessa forma, tem-se como objetivo: correlacionar a gravidade da disfagia com o risco de aspiração e gravidade da doença, investigar o impacto na qualidade de vida de acordo com a gravidade da disfagia e gravidade da doença e avaliar se o estágio da doença e se o risco de aspiração tem impacto na qualidade de vida dos parkinsonianos.

\section{MÉTODOS}

Estudo observacional, com desenho transversal, realizado no período de julho a outubro de 2019 em 24 pacientes diagnosticados com DP atendidos no ambulatório de laringe do serviço de Otorrinolaringologia de um Hospital Universitário vinculado a Universidade Federal do Pará (UFPA), sediado em um município paraense.

O estudo foi iniciado após aprovação do Comitê de Ética em Pesquisa da UFPA - Hospital Universitário João de Barros Barreto da Universidade Federal do Pará (CAAE: 12245919.0.0000.0017) e consentimento da instituição, em consonância com a Resolução 466/2012 do Conselho Nacional de Saúde que regulamenta as questões operacionais e éticas dos trabalhos científicos envolvendo seres humanos. Os pacientes foram orientados quanto aos objetivos e procedimentos da pesquisa, assinando em concordância o Termo de Consentimento Livre e Esclarecido para poderem participar deste estudo.

Os participantes foram avaliados por meio de análise qualitativa e quantitativa do estágio da doença através da escala modificada de estadiamento para sintomas motores de Hoenh e Yahr (Escala H\&Y), da avaliação endoscópica da deglutição para avaliação da gravidade da disfagia, de questionário sobre a qualidade de vida (The 39 item Parkinson's Disease Question - PDQ-39) e de um instrumento de auto avaliação do risco de disfagia e aspiração (Eating Assessment Tool - EAT-10).

A Escala H\&Y foi utilizada para avaliar o estágio da DP tendo como base os sintomas motores, graduando em 5 estágios. Esta avaliação foi realizada por neurologista e geriatra treinados. Caracterizando-os como incapacidade leve os classificados nos estágios 1 a 2; incapacidade moderada os pacientes no estágio 3 e como incapacidade grave os estágios 4 e 5 (SHENKMAN ML, et al., 2001).

Utilizou-se o exame de videonasofaringolaringoscopia da deglutição, também chamado de videoendoscopia da deglutição (VED) para a avaliação da gravidade da disfagia. Para a realização da VED 
foram utilizados os seguintes equipamentos: nasofibroscópio flexível (Olympus 2,7mm), câmera de endoscopia (Olympus HD Camera II CV-180) e fonte de luz (Olympus HD Camera II CV-180) adaptados a televisão (NDS RADIANCE 26"HD).

Durante a VED, conforme protocolo modificado de Santoro PP, et al. (2011), o nasofibroscópio flexivel foi direcionado através da cavidade nasal com menor resistência, sem anestésico tópico, em direção a cavidade oral, faringe e laringe para observação da morfofisiologia. Foi avaliada a sensibilidade através do toque do fibroscópio em estruturas como: base da língua, face laríngea da epiglote, pregas vestibulares e ariepiglóticas sem a presença de alimento. Em seguida, foi fornecido aos pacientes alimentos em três consistências distintas (líquido, pastoso e sólido), corados com azul de metileno, para avaliação da fase faríngea da deglutição. Para a consistência líquida foi utilizado água filtrada, para a pastosa purê de batata sem leite e para o sólido bolacha de água e sal. Ficou padronizado, caso não houvesse contraindicação, o volume de 50 $\mathrm{ml}$ para o líquido, 15 gramas (equivalente a uma colher de sobremesa) para o pastoso e 2 gramas (equivalente a um quarto de uma bolacha água e sal) para o sólido.

Por meio de um questionário padrão pré-determinado pelos autores da pesquisa, segundo dados da VED, classificou-se a a deglutição em: deglutição normal, disfagia leve, moderada e grave. Foi designado deglutição normal quando ocorreram até três tentativas de propulsão para clareamento do bolo alimentar, com ausência de estase alimentar, salivar, penetração e aspiração. Nomeou-se disfagia leve quando houve mais de 3 tentativas para propulsão alimentar sem aspiração ou penetração, disfagia moderada quando ocorreu penetração, mas ainda não houve aspiração e disfagia grave na presença de aspiração (TSUJI DH, 2017; SANTORO PP, et al., 2011).

O PDQ-39 foi utilizado para avaliar a percepção que o paciente tem sobre a sua qualidade de vida (SILVA J, et al., 2011; SOUZA RG, et al., 2007). Ele é composto por 39 questões divididas em 8 domínios (mobilidade, atividades de vida diária, emoção, estigma, suporte social, comunicação, cognição e desconforto corporal). Cada item pode ser respondido segundo cinco respostas pré-determinadas, sendo elas: nunca, raramente, algumas vezes, frequentemente e sempre. A pontuação de cada item varia de zero a cem pontos, onde o zero significa melhor e cem uma pior qualidade de vida (CAROD-ARTAL, 2007). O escore total e de cada domínio para cada indivíduo foi calculado de acordo com a seguinte fórmula:

$$
\frac{\text { soma dos escores de cada questão na dimensão }}{4 \text { ( máx. do escore por questão) X no } \text { de questões na dimensão }^{\circ}} \times 100
$$

No instrumento de auto avaliação da alimentação, EAT-10, buscou-se a auto avaliação da identificação do risco de disfagia e aspiração. Este é composto por dez questões, sendo três do domínio funcional, três do domínio emocional e quatro do domínio físico que podem afetar a vida do parkinsoniano. A pontuação menor ou igual a 3 significa sem risco de disfagia e aspiração. Pontuação maior que 3 nesta escala significa risco para disfagia e aspiração (GONÇALVES MIR, et al., 2013). Os critérios de inclusão adotados foram: pacientes com diagnóstico de Parkinson nos estágios 1 a 4 de acordo com a Escala H\&Y, com ou sem queixa de deglutição e sem outra doença neurológica associada.

Pacientes diagnosticados com DP classificados no estágio 5 segundo a Escala H\&Y foram excluídos uma vez que abrangem pacientes muito debilitados dificultando a realização do exame, bem como a possibilidade de complicações como episódios de aspiração durante a realização do procedimento. Além disso, foram excluídos da pesquisa pacientes que se recusaram a participar da pesquisa ou assinatura do TCLE, sem diagnóstico conclusivo de DP ou que não realizaram todas as avaliações necessárias.

Os dados obtidos foram registrados primeiramente em planilha de Microsoft Excel®, e posteriormente foram exportados para o programa Bioestat $5.3 \AA$. As características epidemiológicas e clínicas foram descritas usando a estatística descritiva (as variáveis categóricas foram apresentadas em frequências e percentuais). O Teste G foi utilizado para avaliar a associação entre auto avaliação alimentar (EAT-10) com a gravidade da disfagia e com o estadiamento da doença (Escala H\&Y). O coeficiente de correlação de Spearman foi utilizado para avaliar o grau de correlação entre o PDQ-39 total e a Escala H\&Y. O nível de significância utilizado foi de $5 \%$. 


\section{RESULTADOS}

A amostra foi composta por 24 pacientes com DP, sendo 18 (75\%) pacientes do sexo masculino e 6 (25\%) pacientes do sexo feminino. A faixa etária desses pacientes foi classificada de acordo com a Organização Mundial de Saúde (OMS) classificando-os em quatro estágios: a meia-idade, que corresponde à faixa etária de 45 a 59 anos; o idoso, pessoas entre 60 e 74 anos; o ancião, indivíduos com 75 até 90 anos; e a velhice extrema, para quem passa dos 90 anos. Observou-se uma maior prevalência $(45,8 \%)$ na faixa etária de 60-74 anos e uma média de idade de 66,5 anos. A idade máxima foi de 81 anos e mínima de 48 anos (Tabela 1).

Tabela 1 - Perfil epidemiológico e tempo de evolução da doença em pacientes portadores de DP atendidos no Hospital Universitário Bettina Ferro de Souza, Belém - PA, no período de julho a outubro de 2019.

\begin{tabular}{|c|c|c|}
\hline & $\begin{array}{l}\text { Frequência } \\
\qquad \mathrm{N}=24\end{array}$ & $\%$ \\
\hline \multicolumn{3}{|l|}{ Sexo } \\
\hline Masculino & 18 & 75.0 \\
\hline Feminino & 6 & 25.0 \\
\hline \multicolumn{3}{|l|}{ Idade } \\
\hline 45-59 anos & 4 & 16.6 \\
\hline $60-74$ anos & 14 & 58,3 \\
\hline $75-90$ anos & 6 & 25.0 \\
\hline$>90$ anos & 0 & 0 \\
\hline \multicolumn{3}{|c|}{ Tempo de evolução } \\
\hline 1 a 3 anos & 5 & 20.8 \\
\hline 4 a 6 anos & 12 & 50.0 \\
\hline 7 a 9 anos & 3 & 12.5 \\
\hline$>10$ anos & 4 & 16.7 \\
\hline \multicolumn{3}{|c|}{ Escala de Hoehn \& Yar } \\
\hline 1 a 1.5 & 8 & 33.3 \\
\hline 2 a 2.5 & 11 & 45.8 \\
\hline 3 & 2 & 8.3 \\
\hline 4 & 3 & 12.5 \\
\hline
\end{tabular}

Fonte: Barbosa ALG, et al., 2019.

Nesses pacientes o tempo médio de diagnóstico da doença foi de 6 anos, apresentando apenas quatro pacientes com tempo maior que 10 anos, com tempo máximo de 15 anos de doença em um paciente. Ao classificá-los na Escala H\&Y, observou-se uma maior prevalência de 45,8\% nos estágios 2 a 2.5, caracterizando-os com uma incapacidade leve a moderada (Tabela 1).

De acordo com os dados coletados por meio da VED, apenas $3(12,5 \%)$ pacientes apresentaram deglutição normal e a disfagia moderada apresentou maior prevalência, presente em $41,7 \%$ dos pacientes (Gráfico 1). 
Gráfico 1 - Distribuição do grau de disfagia em pacientes portadores de DP atendidos no Hospital Universitário Bettina Ferro de Souza, Belém - PA, no período de julho a outubro de 2019.

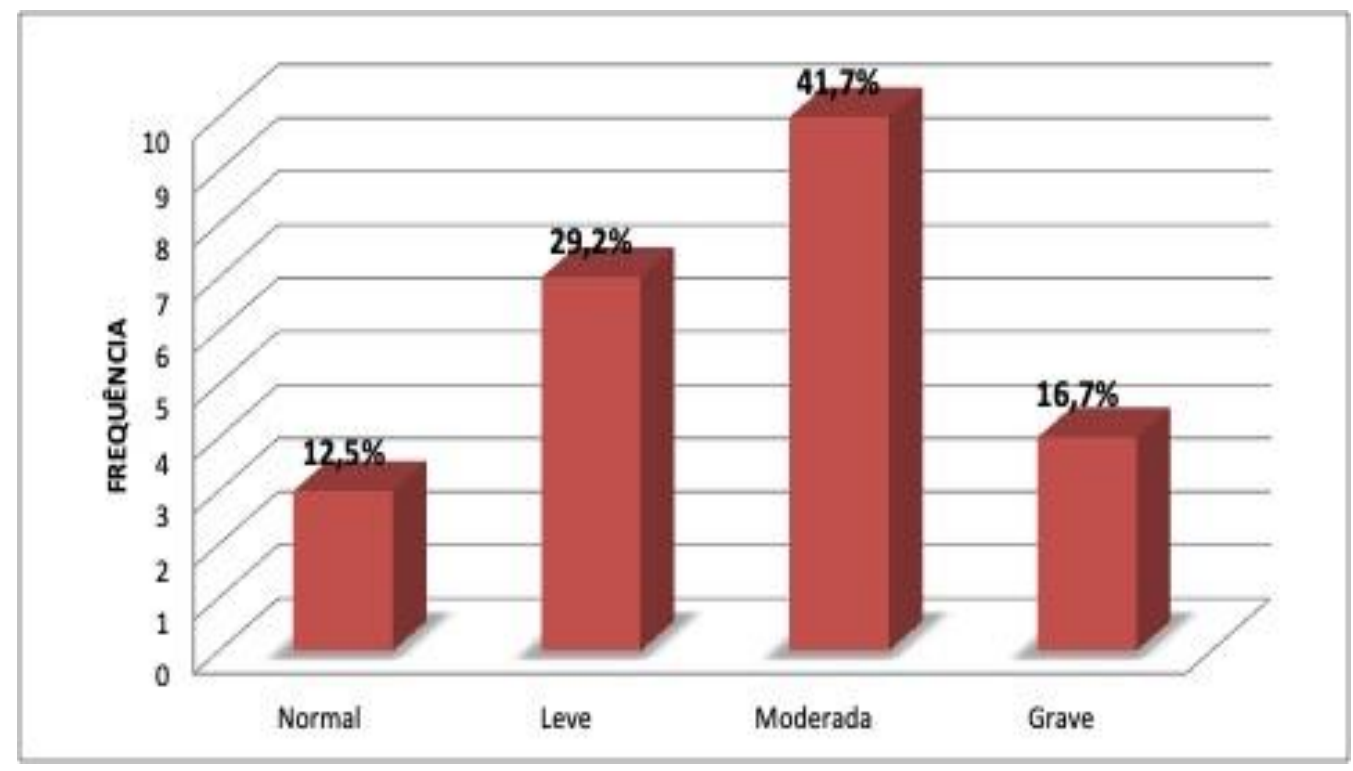

Fonte: Barbosa ALG, et al., 2019.

Todos os pacientes avaliados apresentaram déficit mensurados pelo PDQ-39. Em ordem decrescente de pior qualidade de vida, destacou-se o domínio mobilidade com uma média de 56,6 pontos seguido do domínio no suporte social (55,06 pontos) e desconforto social (50,71 pontos). A melhor pontuação (22,5 pontos) foi observada no domínio de comunicação (Gráfico 2).

Gráfico 2 - Valores máximos, mínimos e a média obtida após a aplicação do PDQ-39 em pacientes portadores de DP atendidos no Hospital Universitário Bettina Ferro de Souza, Belém - PA, no período de julho a outubro de 2019.

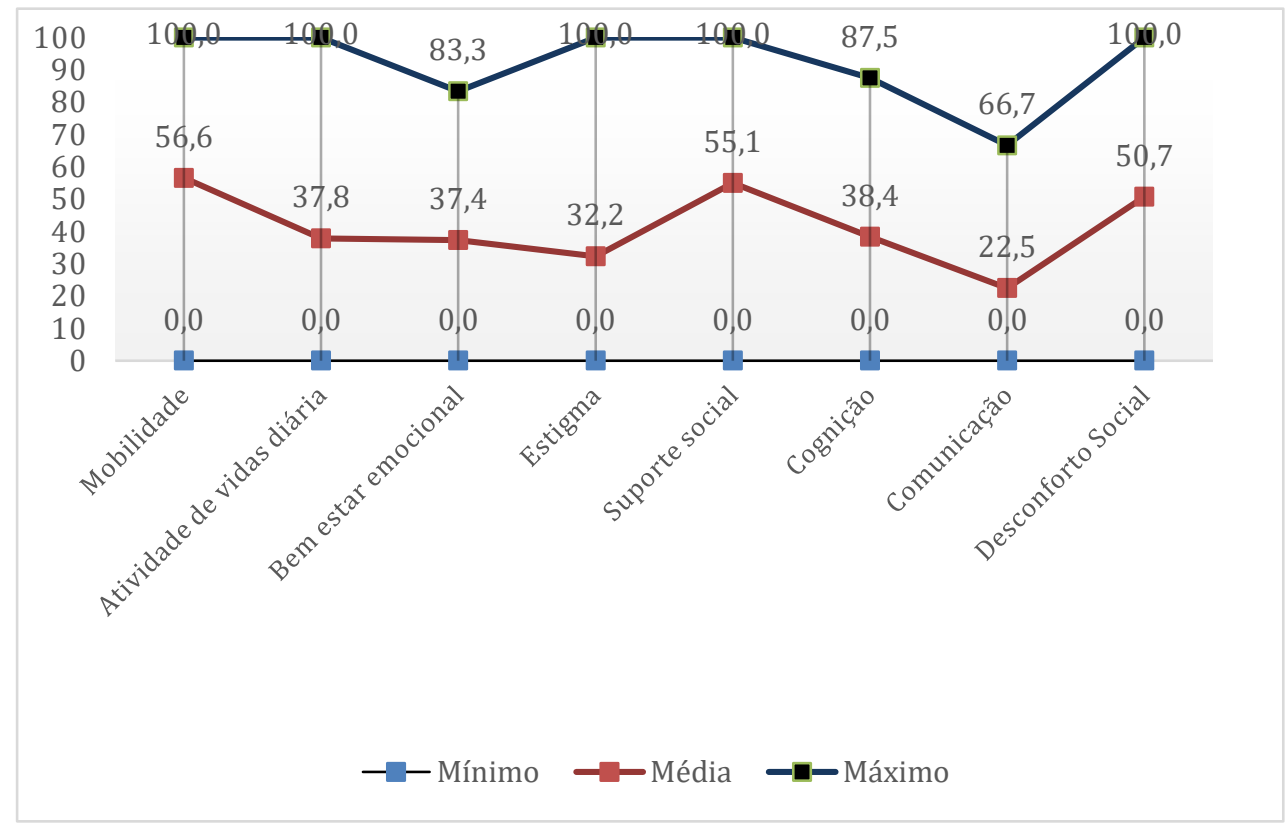

Fonte: Barbosa ALG, et al., 2019. 
Ao comparar a gravidade da doença (Escala H\&Y) com a mediana do escore do PDQ-39 total, observouse uma mediana de 38,2 $\pm 14,9$ em pacientes com Escala H\&Y leve (estágios 1; estágio 1,5 e estágio 2) e $70,8 \pm 14,7$ em pacientes com Escala H\&Y grave (estágio 3 e estágio 4) com uma diferença significativa entre eles $(p<0,05)$. Não foi possível avaliar correlação em pacientes com Escala H\&Y moderado (estágio 2,5) por limitações da amostra (Tabela 2).

Tabela 2 - Comparação entre os parkinsonianos com acometimento leve e grave segundo o PDQ-39 em pacientes portadores de DP atendidos no Hospital Universitário Bettina Ferro de Souza, Belém - PA, no período de julho a outubro de 2019.

\begin{tabular}{ccccc}
\hline \multicolumn{5}{c}{ PDQ-39 Total } \\
\hline Escala H\&Y & Frequência & Mediana & Desvio-quartílico & Valor de $\mathbf{p}$ \\
\hline Leve $^{*}$ & 16 & 38.2 & 14.9 & 0.003 \\
Grave $^{*}$ & 5 & 70.8 & 14.7 &
\end{tabular}

Legenda: Teste de Mann-Whitney; ${ }^{*}$ indica diferença significativa entre os grupos de acometimento leve e grave $(p<0,05)$.

Fonte: Barbosa ALG, et al., 2019.

Ao correlacionar a avaliação perceptual que o paciente tem sobre a sua qualidade de vida (PDQ-39) e o grau de disfagia, observou-se uma pior qualidade de vida no domínio da cognição nos pacientes com disfagia grave (mediana de 59,4 pontos). Nos demais domínios não se observou piora da qualidade de vida com a gravidade da disfagia. De uma forma abrangente (PDQ-39 Total), os pacientes com deglutição normal apresentaram uma mediana de 70,8 pontos; disfagia leve mediana de 41,4 pontos; disfagia moderada de 38,2 pontos e disfagia grave de 59,8 pontos (Tabela 3 ).

Tabela 3 - Correlação entre PDQ-39 e seus domínios individuais e total em relação ao grau de disfagia em pacientes portadores de DP atendidos no Hospital Universitário Bettina Ferro de Souza, Belém - PA, no período de julho a outubro de 2019.

\begin{tabular}{ccc}
\hline PQD-39.6: Domínio Cognição & & \\
\hline Disfagia & Mediana & IC 95\% \\
\hline Normal & 25.0 & $18.8-56.3$ \\
Leve & 31.2 & $8.9-62.3$ \\
Moderada & 28.1 & $18.8-48.8$ \\
Grave & 59.4 & $33.3-87.5$ \\
\hline PQD-39 Total & & \\
\hline Disfagia & Mediana & IC 95\% \\
\hline Normal & 70.8 & $18.5-73.7$ \\
Leve & 41.4 & $20.8-44.1$ \\
Moderada & 38.2 & $28.2-46.4$ \\
Grave & 59.8 & $25.0-74.0$ \\
\hline
\end{tabular}

Fonte: Barbosa ALG et al., 2019.

O questionário do EAT-10 evidenciou risco de aspiração (escore maior que 3) em 16 (66,7\%) dos pacientes, com escore máximo de 22 pontos, não havendo significância estatística entre a gravidade da disfagia e auto avaliação da disfagia pela escala EAT-10 $(p=0,941)$ (Tabela 4). 
Tabela 4 - Correlação entre o grau de disfagia e Eating Assessment Tool (EAT-10) em pacientes portadores de DP atendidos no Hospital Universitário Bettina Ferro de Souza, Belém - PA, no período de julho a outubro de 2019.

\begin{tabular}{|c|c|c|c|c|c|c|c|}
\hline \multicolumn{8}{|c|}{ EAT-10 } \\
\hline Disfagia & $\leq 3$ & $\%$ & $>3$ & $\%$ & Total & $\%$ & Valor de $p$ \\
\hline Normal & 1 & 33.3 & 2 & 66.7 & 3 & 100.0 & \multirow{5}{*}{0.941} \\
\hline Leve & 3 & 42.9 & 4 & 57.1 & 7 & 100.0 & \\
\hline MOD & 3 & 30.0 & 7 & 70.0 & 10 & 100.0 & \\
\hline Grave & 1 & 25.0 & 3 & 75.0 & 4 & 100.0 & \\
\hline Total & 8 & 33.3 & 16 & 66.7 & 24 & 100.0 & \\
\hline
\end{tabular}

Legenda: Teste $\mathrm{G}$.

Fonte: Barbosa ALG et al., 2019.

\section{DISCUSSÃO}

$\mathrm{Na}$ amostra analisada houve uma predominância do sexo masculino. Em estudos realizados nos Estados Unidos, cientistas mostram que, de acordo com revisões literárias de sete artigos dos últimos anos, pelo menos $50 \%$ dos homens estão propensos a desenvolver DP (MILLER et al., 2006). Alguns autores revelam que mulheres correm menos risco de desenvolverem DP porque produzem o estrogênio o que, segundo estes pesquisadores, exerce um efeito protetor para os receptores de dopamina (BASS NH e MORRELL RM, 1992; LUAU LML e BRETELER MMB, 2005).

Estudo de Pringsheim T, et al. (2014) reafirma que os homens tiveram uma prevalência significativamente aumentada de DP, sendo esta de 134 por 100.000 em relação às mulheres, com prevalência de DP de 41 por $100.000(p<0,05)$.

A DP geralmente acomete individuos após 50 anos de idade, com pico aos 60 anos (DIAS et al., 2016; PRINGSHEIM T, et al., 2014), resultado semelhante ao encontrado nesta pesquisa, onde houve uma predominância na faixa etária de 60-69 anos. Aproximadamente $2 \%$ da população mundial com idade superior aos 65 anos são acometidos pela DP, considerada a segunda doença senil mais comum (FUKUNAGA et al., 2014). A DP não é exclusiva de idosos, havendo relatos do desenvolvimento do quadro em indivíduos mais jovens (DIAS et al., 2016; HOEHN, 1976), como observado em um individuo da pesquisa diagnosticado aos 42 anos de idade.

A disfagia ocorre nos portadores de DP em algum momento da evolução da doença devido a rigidez, bradicinesia e incoordenação do mecanismo da deglutição (MONTEIRO D, et al., 2014). Nesses pacientes a prevalência da disfagia pode ser até três vezes maior do que em idosos saudáveis (KWON M e LEE JH, 2019) com mais de $80 \%$ pacientes com DP apresentando disfagia (GAECKLE M, et al., 2019), concordando com nosso estudo onde foi observado disfagia, em diversos graus, em $87,5 \%$ dos paciente.

Há divergência na literatura sobre a relação da gravidade da doença com a severidade da disfagia. Um estudo relatou a disfagia grave aproximadamente 10 a 11 anos após o início dos sintomas (COELHO M, et al., 2010), com um aumento surpreendente após 15 anos de evolução (UMEMOTO G e FURUYA H, 2019). Segundo Kwon M e Lee JH (2019), a duração da doença e sintomas motores não apresenta correlação significativa com os sintomas relacionados ao sistema digestivo, dentre eles a disfagia.

Já Silva J, et al. (2011) refere que a disfagia pode ainda ser o primeiro sinal inicial da doença. No nosso estudo, 70,8\% dos pacientes apresentaram um tempo de evolução de até 6 anos, com predominância de classificação leve na Escala H\&Y (79,1\%). Houve prevalência de 41,7\% de disfagia moderada.

A disfagia é muitas vezes subdiagnosticada, devido a baixa consciência do paciente, condições e subutilização de ferramentas e instrumento objetivos validados para a sua avaliação. A auto avaliação da disfagia, EAT-10, é um exemplo de ferramenta que o presente estudo demostrou não haver correlação com o exame de videoendoscopia de deglutição e com a gravidade da doença, demostrando que a medida subjetiva não foi um bom indicador nesta amostra para detectar a disfagia em pacientes com DP. 
O comprometimento da qualidade de vida nos pacientes com DP acontece muito cedo, podendo demonstrar decréscimo desde os primeiros estágios da doença e piorando com a sua severidade (SCHRAG A, et al., 2000), como mostrado nesse estudo, onde todos os pacientes avaliados pelo PDQ-39 apresentaram deficits mensurados.

O estágio da doença de acordo com a Escala H\&Y é um fator isolado de piora na qualidade de vida nesses individuos (SLAWEK J, et al., 2005; SILVA J, et al., 2011); observando uma piora na qualidade de vida com os estágios mais avançados da doença (PERTERNELLA FMN e MARCON SS, 2012; SOUZA et al., 2007). Neste estudo, houve correlação significativa da piora de qualidade de vida nos estágios mais avançados da doença.

$\mathrm{Na}$ avaliação da qualidade de vida de forma mais abrangente, (PDQ-39 Total) os pacientes sem disfagia apresentaram pior qualidade de vida que os disfágicos. Este dado pode ser sugerir que tarefas motoras, ligadas a interação social e aspectos emocionais podem estar sendo importantes causadores de piora na qualidade de vida em pacientes parkinsonianos sem disfagia.

$\mathrm{Na}$ amostra, a mobilidade, o suporte social e o desconforto social foram os domínios que proporcionaram uma maior percepção de pior qualidade de vida. $\mathrm{Na}$ literatura, outras variáveis parecem interferir significativamente na qualidade de vida desses pacientes. A depressão parece ser o fator mais importante que afeta a qualidade de vida dos pacientes com DP, devendo ser identificada e tratada efetivamente (SLAWEK, J et al., 2005).

Estudos mostram que a qualidade de vida em pacientes com DP é determinada primeiramente pela depressão, e após é influenciada por sintomas físicos somados ao isolamento social e sedentarismo (SILVA J, et al., 2011).

O PDQ-39 compreende oito dimensões ou domínios, sendo um deles a avaliação da depressão de forma indireta pelo dominio de bem-estar emocional, não sendo possivel aferir a sua correlação direta com a qualidade de vida nesse questionário. Além disso, as flutuações do sistema motor, principalmente a instabilidade postural e a incapacidade de resposta com a medicação para a doença de base parecem interferir na qualidade de vida (SCHRAG A, et al., 2000).

Nos estudos de Lana RC, et al. (2007) e Silva J, et al. (2011) observou-se pior percepção da qualidade de vida nas dimensões "Atividade Vida Diária" e "Mobilidade". Esses domínios avaliam tarefas de origem motora, envolvendo situações como andar, realizar atividades de lazer, tomar banho ou vestir-se, sendo tarefas que necessitam de um suporte motor adequado. Tais achados foram corroborados neste estudo, indicando que as dimensões relacionadas aos aspectos físicos da doença são aquelas que o parkinsoniano apresenta uma redução na qualidade de vida, uma vez que os principais sinais e sintomas do DP são sintomas motores, interferindo significamente na qualidade de vida.

Com a progressão da DP, problemas relacionados a memória, alteração da atenção e pensamento podem acontecer de forma mais grave quando comparados com o esperado no envelhecimento normal. Na maioria dos casos, as alterações cognitivas no DP acontecem em fases mais tardias da doença ou com o decorrer do envelhecimento, geralmente depois dos 65 anos (KALLASH P, et al, 2018; GALHARDO MMAMC, et al., 2009).

Vários estudos demonstram que os pacientes parkinsonianos que exibem distúrbios cognitivos são habitualmente mais idosos no início da doença, e estão mais gravemente acometidos. Diante disso, o avanço no comprometimento neurológico evolui com lentidão e falta de controle das estruturas, relacionadas às fases preparatória e oral da deglutição, aumentando a quantidade de resíduos alimentares em valéculas e/ou recessos piriformes, o que acarreta maior risco para a penetração laríngea e/ou aspiração traqueal pósdeglutição (AVIV A, et al, 2005). Pode-se correlacionar a piora no escore do domínio cognitivo em pacientes com disfagia grave relatados neste estudo. Em concordância com estudos de Fabbri M, et al. (2019), a gravidade da disfagia prediz a ocorrência de marcos adicionais da gravidade da doença, sendo por isso importante sua avaliação precoce. 


\section{CONCLUSÃO}

A disfagia é um sintoma comum em pacientes com DP podendo aparecer em qualquer momento da doença, independente da sua gravidade clínica. Detectou-se disfagia em $87,5 \%$ dos pacientes, sendo de grau moderado em $41,7 \%$ deles. Observou-se pior qualidade de vida no domínio da cognição nos pacientes com disfagia grave. Estes achados devem alertar os profissionais de saúde sobre a importância de se investigar e reabilitar precocemente a disfagia em pacientes diagnosticados com DP a fim de reduzir o impacto na qualidade de vida e sobrevida destes pacientes.

\section{REFERÊNCIAS}

1. AVIV A, et al. Flexible endoscopic evaluation of swallowing with sensory testing: patient characteristics and analysis of safety in 1,340 consecutive examinations. Annals of Otology, Rhinology, and Laryngology, v. 114, n.03, p. 173-176, 2005.

2. BASS NH, MORRELL RM. The neurology of swallowing. Dysphagia: diagnosis and management, v. 02, p.1-29, 1992.

3. BELO LR, et al. Surface electromyography of the suprahyoid muscles during deglutition of elderly people without neurological diseases and with Parkinson disease. Revista CEFAC, v. 11, n. 02, p. 268-280, abr-jun 2009.

4. CARNEIRO D, et al. Qualidade de vida em disfagia na doença de Parkinson: uma revisão sistemática. Revista CEFAC (online), vol.15, n.5, p.1347-1356, 2013.

5. CAROD-ARTAL FJ, et al. Independent validation of SCOPA-psychosocial and metric properties of the PDQ-39 Brazilian version. Movement Disorders, 2007.

6. COELHO M, et al. Late-stage Parkinson's disease: the Barcelona and Lisbon cohort. Journal of Neurology, v.257, n. 09, p. 1524-1532, sep 2010.

7. DIAS AE, et al. Speech disorders did not correlate with age at onset of Parkinson's disease. Arquivos de NeuroPsiquiatria. v 47, n. 02, feb 2016.

8. DORSEY ER, et al. Projected number of people with Parkinson disease in the most populous nation, 2005 through 2030. Neurology. v. 68, n 05, p. 384-386, 2007.

9. FABBRI M, et al. Dysphagia predicts poor outcome in late-stage Parkinson's disease. Parkinsonism and related disorders, v. 64, p. 73-81, feb 2019.

10. FUKUNAGA JY, et al. Postural Control In Parkinson's Disease. Brazilian Journal of Otorhinolaryngology. v.80, n 06, dec-2014.

11. GAECKLE M, et al, 2019. Predictors of Penetration-Aspiration in Parkinson's Disease Patients With Dysphagia: $A$ Retrospective Analysis. Annais of otology, rhinology and laryngology, v. 128, n. 08, p. 728-735, aug 2019.

12. GALHARDO MMAMC, et al. Caracterização dos distúrbios cognitivos na doença de parkinson. Revista CEFAC, v.11, p. 251-257, 2009.

13. GASPARIM AZ, et al. Deglutição e tosse nos diferentes graus da doença de Parkinson. Arquivos Internacionais de Otorrinolaringologia / International Archives Otorhinolaryngoly, v.15, n.2, p. 181-188, abr-mai-junho 2011.

14. GONÇALVES MIR, et al. Equivalência da versão brasileira do Eating Assessment Tool - EAT-10. CoDAS, São Paulo, v. 25, n. 6, p. 601-604, 2013.

15. HELY MA, et al. The Sydney multicenter study of Parkinson's disease: the inevitability of dementia at 20 years. Movement Disorders Society, v. 23, n. 06, p. 837-844, apr 2008.

16. HOEHN MM. Age distribution of patients with parkinsonism. Journal of the American Geriatrics Society. v. 24 , n. 02 , p. 79-85, 1976.

17. KALLASH $P$, et al. Consensus Statement on the classification of tremors from the task force on tremor of the International Parkinson and Movement Disorder Society. Movement Disorders. v. 33, n 02, jan 2018.

18. KWON M, LEE JH. Oro-Pharyngeal Dysphagia in Parkinson's Disease and Related Movement Disorders. Journal Of Movement Disorders. v.12, n. 03, p. 152-160, sep 2019.

19. LANA RC, et al. Percepção da qualidade de vida em indivíduos com doença de parkinson através do PDQ-39. Revista Brasileira de Fisioterapia. v.11, n.05, p. 397-402, set-out 2007.

20. LIM A, et al. A pilot study of respiration and swallowing integration in parkinson's disease: "On" and "Off" levodopa. Dysphagia, v. 23, p. 76-81, 2008.

21. LUAU LML, BRETELER MMB. Epidemiology of Parkinson's disease. The Lancet. Neurology. v.05, n. 06, p. 525-535, 2005.

22. MILLER N, et al. Hard to swallow: dysphagia in Parkinson's disease. Age Ageing. V. 25, p. 614-618, 2006. 
23. MONTEIRO D, et al. Relação entre disfagia e tipos clínicos na doença de Parkinson. Revista CEFAC (online), v. 6, n 02, p. 620-627, mar-abr. 2014.

24. PERTERNELLA FMN, MARCON SS. Qualidade de vida de indivíduos com Parkinson e sua relação com tempo de evolução e gravidade da doença. Revista Latino-Americana Enfermagem, v. 20, n. 02, mar-abr 2012.

25. PRINGSHEIM T, et al. The prevalence of Parkinson's disease: A systematic review and meta-analysis. Movement Disorders, v. 29, n.13, p. 1583-1590, 2014

26. SANTORO PP, et al. Otolaryngology and speech therapy evaluation in the assessment of oropharyngeal dysphagia: a combined protocol proposal. Brazilian Journal of Otorhinolaryngology. v. 77, n. 02, p. 201-213, mar-april 2011.

27. SANTOS HIV. Limite de disfagia e capacidade de deglutição fracionada em indivíduos com doença de Parkinson. Lisboa, 2017. Dissertação (Mestrado em terapia da fala na especialidade de motricidade orofacial e deglutição), Universidade de Lisboa, 2017.

28. SCHRAG A, et al. What contributes to quality of life in patients with Parkinson's disease? Journal Of Neurology, Neurosurgery And Psychiatry. v. 69, n. 03, p. 308-312, sep 2000.

29. SHENKMAN ML, et al. Spinal movement and performance of standing reach task in participants with and without Parkinson disease. Physical Therapy, vol. 81, p. 1400-1411, 2001.

30. SILVA J, et al. Mensuração da qualidade de vida de indivíduos com a doença de Parkinson por meio do questionário PDQ-39. Fisioterapia em Movimento. v.24, n.01, p. 141-146, jan/mar 2011.

31. SLAWEK J, et al. Factors affecting the quality of life of patients with idiopathic Parkinson's disease--a cross-sectional study in an outpatient clinic attendees. Parkinsonism and related disorders. v.11, n. 07, p. 465-468, sep 2005.

32. SOUZA RG, et al. Escala para qualidade de vida na doença de Parkinson - PDQ 39 (versão do Português falado no Brasil) como instrumento para avaliação de pacientes com e sem flutuação motora decorrente da levodopa. Arquivos de Neuro-Psiquiatria, v. 65, n. 03, sep 2007.

33. SUTTRUP I, WARNECKE T. Dysphagia in Parkinson's Disease. Dysphagia. v. 31, n 01, p. 24-32, 2015.

34. TSUJI DH. Videonasolaringoscopia: Fundamentos teóricos - Aplicação Prática - Protocolos de Avaliação. 1.ed. Rio de Janeiro: Thieme Revinter Publicações, 2017.

35. UMEMOTO G, FURUYA H. Management of Dysphagia in Patients with Parkinson's Disease and Related Disorders. Internal Medicine, 2019. 